\title{
Efficacy and safety of inhaled a1-antitrypsin in patients with severe $\alpha 1$-antitrypsin deficiency and frequent exacerbations of COPD
}

\author{
Jan Stolk ${ }^{1}$, Naveh Tov ${ }^{2}$, Kenneth R. Chapman $\mathbb{1}^{3}$, Pablo Fernandez ${ }^{4}$, \\ William MacNee ${ }^{5}$, Nicholas S. Hopkinson $\mathbb{1}^{6}$, Eeva Piitulainen ${ }^{7}$, \\ Niels Seersholm ${ }^{8}$, Claus F. Vogelmeier ${ }^{9}$, Robert Bals ${ }^{10}{ }^{10}$, Gerry McElvaney ${ }^{11}$ \\ and Robert A. Stockley ${ }^{12}$
}

\begin{abstract}
Affiliations: ${ }^{1}$ Dept of Pulmonology, Member of European Reference Network LUNG, Leiden University Medical Centre, Leiden, NL. ${ }^{2}$ Dept of Clinical Development, Kamada Ltd, Rehovot, Israel. ${ }^{3}$ Dept of Medicine, University of Toronto, Toronto, ON, Canada. ${ }^{4}$ Independent Consultant, Penn, UK. ${ }^{5}$ University of Edinburgh, Edinburgh, UK. ${ }^{6}$ National Heart and Lung Institute, Imperial College London, London, UK. ${ }^{7}$ Dept of Respiratory Medicine and Allergology, Malmö, Skane University Hospital, Lund University, Lund, Sweden. ${ }^{8}$ Dept of Respiratory Medicine Y, Gentofte University Hospital, Copenhagen, Denmark. ${ }^{9}$ Dept of Medicine, Pulmonary and Critical Care Medicine, Member of the German Center for Lung Diseases (DZL), University Medical Centre Giessen and Marburg, Philipps-University Marburg, Marburg, Germany. ${ }^{10}$ Internal Medicine V - Pulmonology, Member of the German Center for Lung Diseases (DZL), Hospital of the University of the Saarland, Homburg, Germany. ${ }^{11}$ Dept of Respiratory Medicine, Beaumont Hospital, Royal College of Surgeons in Ireland, Dublin, Ireland. ${ }^{12}$ Queen Elizabeth Hospital, Birmingham, UK.
\end{abstract}

Correspondence: Jan Stolk, Dept of Pulmonology, Member of European Reference Network LUNG, Leiden University Medical Centre, Leiden, The Netherlands. E-mail: j.stolkalumc.nl

@ERSpublications

Inhaled $\alpha 1$-antitrypsin did not significantly reduce the time to first exacerbation in patients with severe $\alpha 1$-antitrypsin deficiency who experience frequent exacerbations of COPD in a randomised placebo-controlled clinical trial of 1 year http://bit.ly/2P $5 \mathrm{zXdK}$

Cite this article as: Stolk J, Tov N, Chapman KR, et al. Efficacy and safety of inhaled $\alpha 1$-antitrypsin in patients with severe $\alpha 1$-antitrypsin deficiency and frequent exacerbations of COPD. Eur Respir J 2019; 54: 1900673 [https://doi.org/10.1183/13993003.00673-2019].

ABSTRACT Patients with inherited $\alpha 1$-antitrypsin (AAT) deficiency (ZZ-AATD) and severe chronic obstructive pulmonary disease (COPD) frequently experience exacerbations. We postulated that inhalation of nebulised AAT would be an effective treatment.

We randomly assigned 168 patients to receive twice-daily inhalations of $80 \mathrm{mg}$ AAT solution or placebo for 50 weeks. Patients used an electronic diary to capture exacerbations. The primary endpoint was time from randomisation to the first event-based exacerbation. Secondary endpoints included change in the nature of the exacerbation as defined by the Anthonisen criteria. Safety was also assessed.

Time to first moderate or severe exacerbation was a median of 112 days (interquartile range (IQR) 40-211 days) for AAT and 140 days (IQR 72-142 days) for placebo ( $\mathrm{p}=0.0952)$. The mean yearly rate of all exacerbations was 3.12 in the AAT-treated group and 2.67 in the placebo group $(\mathrm{p}=0.31)$. More patients receiving AAT reported treatment-related treatment-emergent adverse events compared to placebo ( $57.5 \%$ versus $46.9 \%$, respectively) and they were more likely to withdraw from the study. After the first year of the study, when modifications to the handling of the nebuliser were introduced, the rate of safety events in the AAT-treated group dropped to that of the placebo group.

We conclude that in AATD patients with severe COPD and frequent exacerbations, AAT inhalation for 50 weeks showed no effect on time to first exacerbation but may have changed the pattern of the episodes. 


\section{Introduction}

Individuals with severe deficiency of $\alpha 1$-antitrypsin (AAT) due to a Glu ${ }^{342}$ Lys point mutation (ZZ-AATD) are at increased risk of developing chronic obstructive pulmonary disease (COPD) with a dominant emphysema phenotype [1]. They also tend to have an accelerated disease course, with worsening airflow limitation and decreasing gas transfer compared to COPD patients with normal levels of AAT. Airflow limitation in particular is strongly associated with symptoms [2].

These patients frequently experience exacerbations of respiratory symptoms that require steroid and/or antibiotic treatment and may lead to hospital admission. Exacerbations are associated with poor health [3], and have been shown to accelerate the inevitable decline in lung function seen in COPD [4]. A recent multicentre study revealed that increased dyspnoea is the most important component of the Anthonisen criteria driving treatment of an exacerbation [5-7].

The usual standard of care for ZZ-AATD patients with COPD and frequent exacerbations is dual bronchodilator therapy with inhaled corticosteroids. In a number of countries, intravenous (i.v.) AAT is administered to protect against the inflammatory destruction of lung parenchyma and to reduce the development of emphysema [8]. The effect of i.v. augmentation by AAT infusions in ZZ-AATD COPD patients has been studied in three randomised clinical trials, but none demonstrated a statistically significant reduction in the rate or severity of exacerbations [9]. In 2015, the RAPID trial showed a statistically significant slower decline in lung density but no effect on lung function. In addition, there was no significant effect on COPD events (AAT versus placebo, annualised incidence rate of 0.63 versus 0.36 , respectively) [10]. The lack of effect on exacerbations in the RAPID trial may have been partly methodological; it could be explained by a failure to enrich the study population with exacerbation-prone patients and the lack of contemporaneous diary card monitoring to detect exacerbation events. However, it is also possible that the levels of AAT reaching the airway side by the i.v. route are insufficient to modify airway inflammation.

Direct delivery of AAT to the lung by nebulisation might have a higher impact on airway inflammation, but the possible local anti-inflammatory effects of inhaled AAT on the airways or the effect on exacerbations have not yet been explored.

We postulated that inhalation of nebulised AAT could be an effective treatment in ZZ-AATD patients with severe COPD who are at particular risk for exacerbations and whose episodes are not adequately controlled with other inhaled medications.

\section{Methods}

Patients and study design

The NCT01217671 study was a multicentre, double-blind, randomised parallel group, 50-week placebo-controlled trial, conducted at 12 sites between January 2010 and November 2014. After ethical approval of the study by local ethics boards, informed consent was obtained from men and women aged $>18$ years with a diagnosis of emphysema confirmed by computed tomography (CT) scan and documented severe AAT deficiency (ZZ or other rare genotypes with a serum level $<11 \mu \mathrm{M}$ ).

The inclusion criteria included post-bronchodilator forced expiratory volume in $1 \mathrm{~s}$ (FEV1) $<80 \%$ predicted and $\mathrm{FEV} 1 /$ forced vital capacity $(\mathrm{FVC})<70 \%$ predicted [11], and a history of two or more moderate or severe exacerbations requiring a change in treatment (antibiotics, systemic steroids and/or hospitalisation) over the previous 18 months. At least one episode was required to be within the 12 months prior to screening. Patients were allowed any relevant concomitant medications considered to be necessary to treat their condition. Exclusion criteria included immunoglobulin A deficiency, a history of life-threatening allergy or anaphylactic reactions, previous or planned lung transplantation and active smoking during the previous 12 months. After providing signed informed consent, all eligible patients were trained in the handling of the eFlow ${ }^{\circledast}$ inhalation device (PARI Pharma GmbH, Gräfelfing, Germany) and the use of an electronic diary (eDiary), the LogPad (PHT Corporation, Geneva, Switzerland).

\section{Randomisation and treatment}

Eligible patients were randomised 1:1 to receive a twice-daily inhalation of human plasma-derived AAT ( $80 \mathrm{mg}$ in $4 \mathrm{~mL}$ of high-purity liquid ready to use in sterile, single-use glass vials) or $4 \mathrm{~mL}$ of PBS with a

This article has supplementary material available from erj.ersjournals.com

This study is registered as clinical trial NCT01217671. Readers may request the data from N. Tov, who is a co-author of this manuscript.

Received: 21 May 2018 | Accepted after revision: 13 Aug 2019 
similar appearance to the AAT. The randomisation was by a dedicated interactive voice response system prepared by inVentiv Health Clinical Ltd and was stratified by site and by whether the patients were receiving concomitant i.v. AAT infusions (only German sites, constituting no more than $10 \%$ of the study population).

Treatment began at the baseline/randomisation visit if patients had no exacerbation in the previous 4 weeks and treatment continued for a period of 50 weeks. They continued regular bronchodilator therapy, which was administered prior to inhalation of the study drug. Patients were instructed to clean and disinfect the eFlow ${ }^{\circledR}$ inhalation device daily. Each nebulisation session lasted for $\sim 8-15$ min. During the first year, patient feedback included a prolonged nebulisation time, which was resolved by an improvement in the handling of AAT and nebuliser. The mean proportion of vials used during the study was $92 \%$ in the AAT arm and $94 \%$ in the placebo arm. After the study end it was revealed that two patients randomised to placebo mistakenly received inhaled AAT from the pharmacy department of the study site (for 1.5 days and 6.5 days); their data were analysed in the safety population as AAT treated.

\section{Monitoring and treatment of COPD exacerbations}

The eDiary consisted of daily questions for the patient designed to evaluate any change in symptoms (breathlessness (dyspnoea) and sputum volume and colour), well-being, concomitant medications and hospitalisations [12]. The diary card data were then automatically transmitted to a secure Internet database via a transmission device. Patients were reminded to fill in the diary by a daily electronic signal. This daily update enabled close monitoring of the patient's condition and identification of the start and end of an exacerbation, defined as an increase in symptoms for at least 2 days with a return to normal for at least 2 days. Details of this monitoring method have been published previously [5]. Because most study patients lived a long distance from the study centre, the treatment of exacerbation episodes was not prescribed by investigators but predominantly left to local medical practitioners.

A dedicated committee, blinded to the treatment allocation, adjudicated all exacerbations by reconciling the eDiary data with the clinical database (treatment use for exacerbations and no other indications), and graded exacerbations from 1 to 3 according to the Anthonisen criteria [7]. A moderate event-based exacerbation was defined as a symptom changes for at least 2 days requiring a course of treatment with antibiotics and/or oral corticosteroids, and a severe exacerbation as an episode with symptom changes for more than 2 days requiring hospitalisation. For patients taking routine antibiotics, any increase in the current dose of antibiotics or change of the type of antibiotics was deemed to indicate a moderate exacerbation.

Additional study procedures are described in the supplementary material.

\section{Statistical analysis}

The primary outcome, time to first moderate/severe exacerbation, was analysed according to the intention-to-treat principle by log-rank test for time to first moderate/severe exacerbation stratified by site. Sensitivity analysis for the primary outcome included subgroups of patients excluding outliers.

The initial aim was to recruit 200 patients, based on the assumption that a clinically meaningful difference of $60 \%$ (placebo group) or $40 \%$ (AAT-treated group) in moderate or severe exacerbations would require at least 92 events to provide $80 \%$ power for a two-sided log-rank test for equality of survival curves at 1 year (a constant hazard ratio of 0.557 ). Following a blinded review of the number of exacerbation events during the study, the statistical requirement for $80 \%$ power to distinguish between the groups was reached after 168 patients had been randomised to study treatment. Sample size calculations indicated that enrolment could be ceased at that point without compromising the power of the primary endpoint.

Table 1 details the demographic and baseline characteristics of patients. Imbalance between treatment groups was tested using ANOVA for continuous variables and Fisher's exact test for categorical variables.

The FEV1 and related spirometry results were measured per protocol as safety parameters.

For the AAT-treated group, any association of safety parameters (adverse events, suspected hypersensitivity, serious adverse events and drug withdrawal) with AAT antibodies status was assessed using rates per 100 days, relative rates and $95 \%$ confidence intervals.

Two-sided tests were used in this study and p-values of $<0.05$ were deemed statistically significant in comparisons of efficacy and safety data. Statistical analyses were done with SAS software (version 9.2 or later; SAS Institute Inc., Cary, NC, USA).

\section{Results}

A total of 210 patients were screened, of whom 168 were randomised to receive either AAT for inhalation (85 patients) or placebo (83 patients). The Consolidated Standards of Reporting Trials (CONSORT) diagram for the study is shown in figure 1 and the demographics of the baseline population are shown in table 1. 
TABLE 1 Demographic and baseline characteristics for intention-to-treat population

\begin{tabular}{|c|c|c|c|}
\hline & \multicolumn{2}{|c|}{ Treatment group } & \multirow[t]{2}{*}{ p-value } \\
\hline & AAT & Placebo & \\
\hline Subjects $\mathrm{n}$ & 85 & 83 & \\
\hline \multicolumn{4}{|l|}{ Age years } \\
\hline Mean \pm SD & $56.5 \pm 9.9$ & $54.4 \pm 10.3$ & 0.1942 \\
\hline Median (IQR) & $57(36-78)$ & $53(37-80)$ & \\
\hline Age $\geqslant 60$ years & $38(44.7)$ & $26(31.3)$ & 0.0824 \\
\hline \multicolumn{4}{|l|}{ Sex } \\
\hline Male & $51(60.0)$ & $49(59.0)$ & 0.9999 \\
\hline Female & $34(40.0)$ & $34(41.0)$ & 0.9999 \\
\hline \multicolumn{4}{|l|}{$\mathrm{BMI} \mathrm{kg} \cdot \mathrm{cm}^{-2}$} \\
\hline Mean \pm SD & $25.8 \pm 4.6$ & $26.3 \pm 5.5$ & 0.5462 \\
\hline Median (IQR) & $24.8(16.8-43.7)$ & $25.6(17.2-58.6)$ & \\
\hline $\mathrm{BMI}<20 \mathrm{~kg} \cdot \mathrm{m}^{-2}$ & $8(9.4)$ & $4(4.8)$ & 0.3702 \\
\hline \multicolumn{4}{|l|}{ FEV1 L } \\
\hline Mean \pm SD & $1.32 \pm 0.49$ & $1.33 \pm 0.53$ & 0.7307 \\
\hline Median (IQR) & $1.12(0.54-2.87)$ & $1.24(0.52-3.48)$ & \\
\hline \multicolumn{4}{|l|}{ FEV $1 \%$ predicted } \\
\hline Mean $\pm S D$ & $42.8 \pm 14.8$ & $41.8 \pm 14.7$ & 0.6617 \\
\hline Median (IQR) & $39.0(24.0-78.0)$ & $39.0(20.0-89.0)$ & \\
\hline \multicolumn{4}{|l|}{ FEV1 group by GOLD stage } \\
\hline $1+\| \mid\left(F^{2} V_{1}>50 \%\right.$ pred $)$ & 22 (25.9) & $13(15.7)$ & $0.1290^{\S \S}$ \\
\hline III+IV (FEV $1 \leqslant 50 \%$ pred) & $63(74.1)$ & 70 (84.3) & \\
\hline \multicolumn{4}{|l|}{ DLco $\%$ predicted } \\
\hline Mean $\pm S D$ & $50.2 \pm 18.0$ & $52.9 \pm 19.8$ & 0.3633 \\
\hline Median (IQR) & $49.6(12.9-135.9)$ & $52.7(14.9-109.6)$ & \\
\hline $\begin{array}{l}\text { Patients with at least two exacerbations } \\
\text { in the } 12 \text { months prior to randomisation }\end{array}$ & $64(75.3)$ & $63(75.9)$ & $1.0000^{\S \S}$ \\
\hline \multicolumn{4}{|l|}{ SGRQ total score ${ }^{+}$} \\
\hline Mean \pm SD & $50.2 \pm 15.0$ & $53.5 \pm 14.5$ & 0.1594 \\
\hline Median (IQR) & $48.6(13.9-90.3)$ & $54.3(19.2-86.4)$ & \\
\hline \multicolumn{4}{|l|}{ SGRQ impact domain score ${ }^{\S}$} \\
\hline Mean $\pm S D$ & $37.3 \pm 17.3$ & $41.2 \pm 16.7$ & 0.1401 \\
\hline Median (IQR) & $34.8(1.6-83.7)$ & $40.9(6.7-80.9)$ & \\
\hline \multicolumn{4}{|l|}{ SGRQ activity domain score ${ }^{f}$} \\
\hline Mean $\pm S D$ & $72.4 \pm 17.7$ & $75.4 \pm 17.3$ & 0.2706 \\
\hline Median (IQR) & $73.0(18.5-100.0)$ & $79.2(17.4-100.0)$ & \\
\hline \multicolumn{4}{|l|}{ SGRQ symptoms domain score ${ }^{\# \#}$} \\
\hline Mean \pm SD & $49.8 \pm 18.8$ & $52.2 \pm 21.2$ & 0.4324 \\
\hline Median (IQR) & $50.3(2.6-100.0)$ & $51.7(0.0-97.6)$ & \\
\hline i.v. AAT-treated patients ${ }^{\text {กा }}$ & $6(7.1)$ & $6(7.2)$ & 0.9999 \\
\hline \multicolumn{4}{|l|}{ Concomitant medication } \\
\hline LAMA & 74 (87) & $69(83)$ & 0.5208 \\
\hline LABA+ICS & $69(81)$ & $69(83)$ & 0.8411 \\
\hline Antibiotic prophylaxis & $7(8.2)$ & $8(9.6)$ & $0.7925^{\S \S}$ \\
\hline Oxygen users at study entry & $9(10.6)$ & $6(7.2)$ & $0.5903^{\S \S}$ \\
\hline
\end{tabular}

Data are presented as $\mathrm{n}(\%)$ if not otherwise indicated. AAT: $\alpha 1$-antitrypsin; IQR: interquartile range; BMI: body mass index; FEV1: forced expiratory volume in 1 s; GOLD: Global Initiative for Chronic Obstructive Lung Disease; DLCo: diffusing capacity of the lung for carbon monoxide; SGRQ: St George's Respiratory Questionnaire; i.v.: intravenous; LAMA: long-acting muscarinic antagonist; LABA: long-acting $\beta 2$ agonist; ICS: inhaled corticosteroid. " : p-values indicate the likelihood of any difference between the two groups at baseline; " : DLCo corrected for haemoglobin and normalised to healthy subjects [13]; ${ }^{+}$: AAT-treated $n=83$, placebo $n=80 ;{ }^{\S}$ : AAT-treated $n=85$, placebo $n=81 ;{ }^{f}$ : AAT-treated $n=84$, placebo $n=82 ;{ }^{\text {\#\# }}$ : AAT-treated $n=84$, placebo $n=83$; กा: only in German sites, patients were treated by i.v. AAT combined with inhaled AAT/ placebo; ${ }^{\S}$ : two-tailed p-value calculated by Fisher's exact test.

\section{Exacerbations}

The primary endpoint of the analysis, time to first moderate/severe exacerbation (event-based definition), was not different between the AAT-treated (median 112 days, IQR 40-211 days) and placebo groups (median 140 days, IQR 72-142 days; $\mathrm{p}=0.0952$ ) (figure 2). There were two severe exacerbations in both the AAT and the placebo groups. 


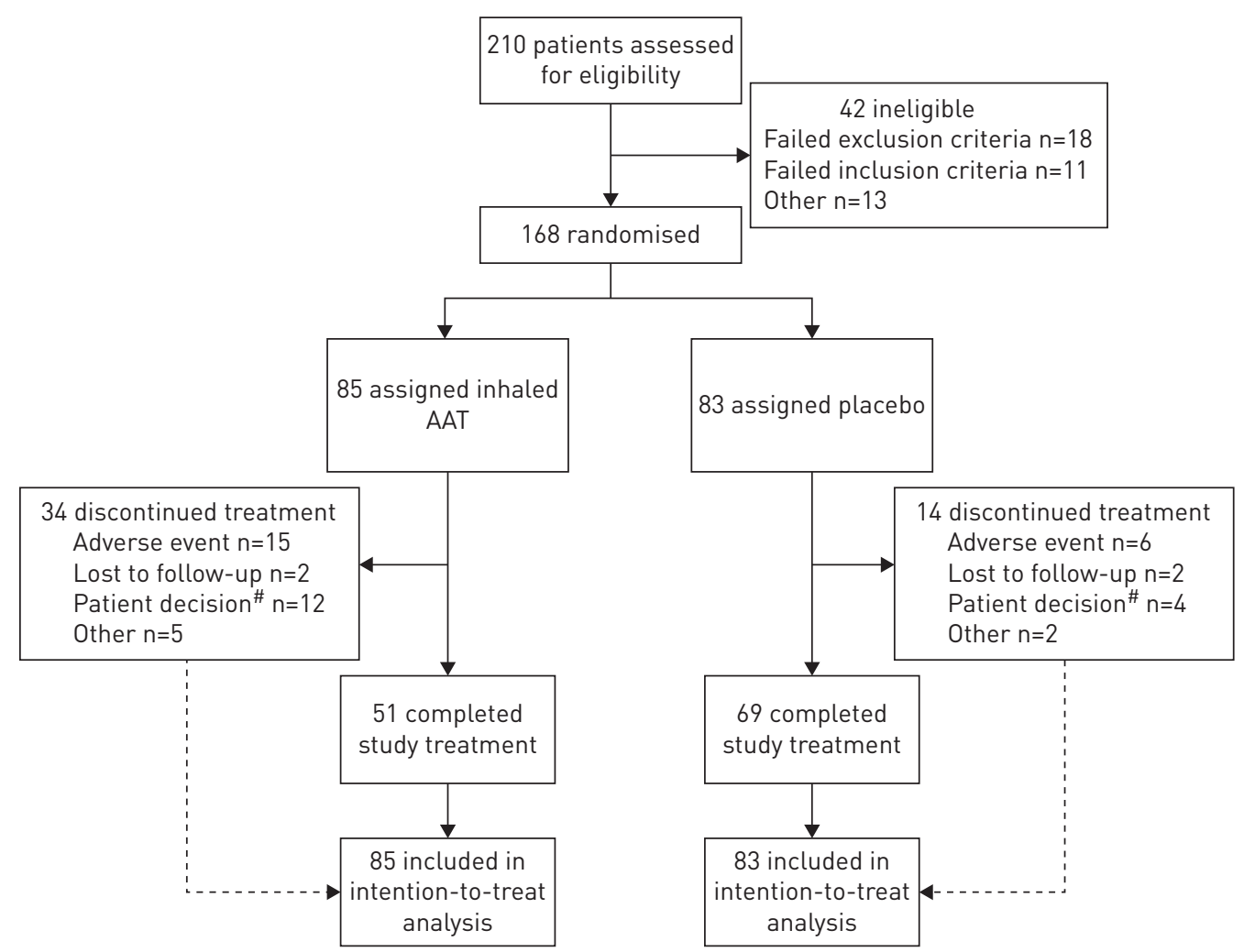

FIGURE 1 Consolidated Standards of Reporting Trials (CONSORT) diagram for intention-to-treat population. AAT: $\alpha 1$-antitrypsin. ${ }^{\#}$ : patient decision not related to adverse event.

After a sensitivity analysis that focused on a less morbid subgroup (excluding those aged $\geqslant 60$ years, with a body mass index $<20 \mathrm{~kg} \cdot \mathrm{m}^{-2}$ and/or with oxygen use; table 1), the median time to first moderate/severe exacerbation was 112 days (IQR 72-146 days) for the AAT-treated group and 126 days (IQR 68-194 days) for the placebo group $(\mathrm{p}=0.1785)$. Analysis of event-based exacerbations ( $\mathrm{mild} / \mathrm{moderate} /$ severe) per

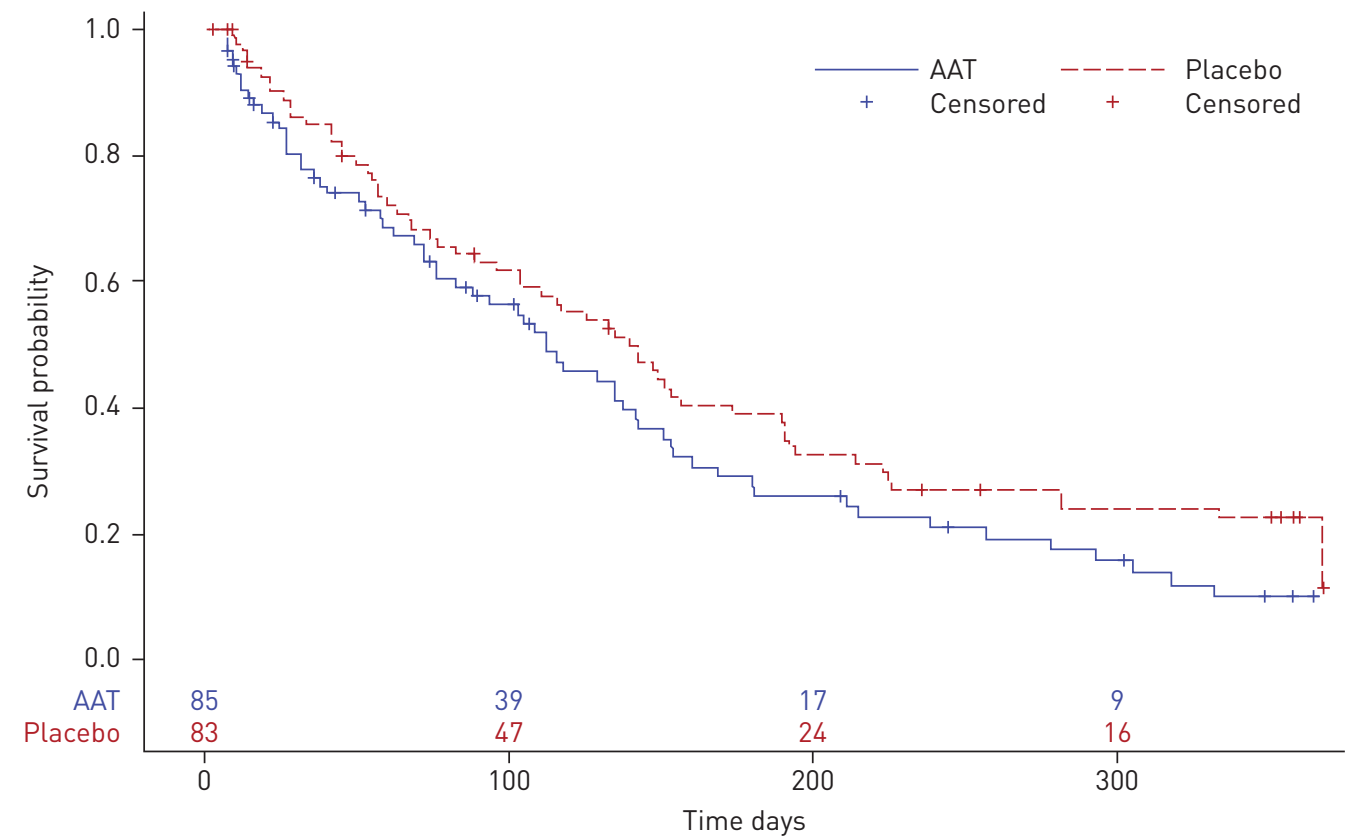

FIGURE 2 Kaplan-Meier analysis of time to first moderate/severe exacerbation. Red and blue numbers denote the number of patients at risk. AAT: $\alpha 1$-antitrypsin. 
patient during the study period revealed 2.26 \pm 2.19 (mean \pm SD) exacerbations in the AAT-treated population and $2.35 \pm 2.38$ in the placebo population (supplementary tables S1 and S2). The mean yearly rates of exacerbations in the AAT and placebo groups were 3.12 and $2.67(\mathrm{p}=0.312)$, respectively.

Despite the lack of difference in time to exacerbation, the AAT-treated group had a lower proportion of first symptom-based exacerbation categorised as Anthonisen's Type I (three symptoms worsening, table 2), at $18.8 \%$ of AAT-treated patients versus $31.3 \%$ of placebo patients $(\mathrm{p}=0.06)$. In parallel, the AAT-treated group had a higher proportion of Type II episodes (two symptoms worsening), at $27.1 \%$ of AAT-treated patients versus $14.5 \%$ of placebo patients $(\mathrm{p}=0.04)$.

Table 2 also shows that for any type of first exacerbation defined by Anthonisen criteria and any combination of deteriorating symptoms, the number of exacerbations treated with a course of oral steroids and/or antibiotics (shown both in the AAT-treated and placebo groups) was lower than the number of exacerbations detected. For example, there were 25 patients in the AAT-treated group with a first Type III exacerbation, of whom only eight received treatment (32\%), while 10 of 27 patients in the placebo group (37\%) received treatment. Also remarkable was that six patients $(23 \%)$ in the placebo group were not treated for a Type I exacerbation (three symptoms deteriorating for more than 2 days) versus three patients $(18 \%)$ in the AAT-treated group. The event counts and rates of event-based exacerbations during the 50 weeks of study are given in supplementary table S2.

\section{Adverse events}

A higher proportion of patients in the AAT-treated group reported treatment-related treatment-emergent adverse events (TEAEs) compared to placebo (57.5\% versus $46.9 \%$, respectively), and $6.9 \%$ of patients in the AAT-treated group reported treatment-related serious adverse events (SAEs) compared to none in the placebo group. Of note, symptoms that classify as Anthonisen criteria were also reported as TEAEs. 15 patients (17.2\%) receiving AAT reported TEAEs that led to treatment discontinuation, compared to six patients $(7.4 \%)$ in the placebo group. Further details about hospitalisations and systemic use of antibiotics and steroids are given in the supplementary material. The most prevalent TEAEs are listed in table 3. The most common TEAEs in both arms were dyspnoea and cough, which had similar rates in the two groups.

Safety events were related to severity of disease. Patients with less severe disease (FEV1>50\% and St George's Respiratory Questionnaire (SGRQ) score <50) had a comparable profile to similarly scoring patients in the placebo group.

After the first year, when the handling of the nebuliser and drug was improved, the rate per 100 days of safety events in the AAT-treated group dropped significantly (from 0.29 to 0.11 withdrawals, 0.27 to 0.15 SAEs and 0.81 to 0.64 exacerbations), becoming similar to that of the placebo group. Moderate exacerbations were balanced between the AAT-treated and placebo groups throughout the study, and exhibited a clear seasonality effect. Withdrawals were imbalanced in favour of placebo, with a lower rate per year after the first year (supplementary figure S4).

\begin{tabular}{|c|c|c|c|c|}
\hline \multirow{2}{*}{$\begin{array}{l}\text { Severity of the first exacerbation by } \\
\text { Anthonisen type }\end{array}$} & \multicolumn{2}{|c|}{ AAT $^{\#}$} & \multicolumn{2}{|c|}{ Placebon } \\
\hline & All & $\begin{array}{c}\text { Treated } \\
\text { exacerbations } \\
\text { (event-based) }\end{array}$ & All & $\begin{array}{c}\text { Treated } \\
\text { exacerbations } \\
\text { (event-based) }\end{array}$ \\
\hline None & $12(14.1)$ & - & 12 (14.5) & - \\
\hline All Type III ${ }^{+}$ & $34(40.0)$ & $10(29.4)$ & 33 (39.8) & $11(33.3)$ \\
\hline Dyspnoea & 25 (73.5) & $8(80.0)$ & 27 (81.8) & 10 (90.9) \\
\hline Sputum volume & $3(8.8)$ & $1(10.0)$ & $5(15.2)$ & $1(9.1)$ \\
\hline Sputum colour & $6(17.6)$ & $1(10.0)$ & $1(3.0)$ & $0(0.0)$ \\
\hline All Type II ${ }^{\S}$ & $23(27.1)$ & $12(52.2)$ & $12(14.5)$ & $4(33.3)$ \\
\hline Dyspnoea+sputum volume & $6(26.1)$ & $5(41.7)$ & $2(16.7)$ & $1(25.0)$ \\
\hline Dyspnoea+sputum colour & $11(47.8)$ & $6(50.0)$ & 4 (33.3) & $2(50.0)$ \\
\hline Sputum volume+sputum colour & $6(26.1)$ & $1(8.3)$ & $6(50.0)$ & $1(25.0)$ \\
\hline Type I ${ }^{f}$ & $16(18.8)$ & 13 (81.3) & $26(31.3)$ & $20(76.9)$ \\
\hline
\end{tabular}

Data are presented as $\mathrm{n}(\%)$. Percentages are reported based on the total number of patients in the Anthonisen class. A patient with more than one specific treatment-emergent adverse event was counted once for that class. ${ }^{\#}: \mathrm{n}=85 ;{ }^{\uparrow}: \mathrm{n}=83 ;{ }^{+}$: one symptom deteriorated; ${ }^{\S}$ : two symptoms deteriorated; ${ }^{f}$ : all three major symptoms deteriorated. AAT: $\alpha 1$-antitrypsin. 


\begin{tabular}{|c|c|c|c|c|}
\hline & \multicolumn{2}{|c|}{ AAT $^{\#}$} & \multicolumn{2}{|c|}{ Placebo ๆ } \\
\hline & Patients & Events & Patients & Events \\
\hline Any TEAE & $86(98.9 \%)$ & $726(43.22)$ & $78(96.3 \%)$ & 615 (32.49) \\
\hline Respiratory, thoracic and mediastinal disorders & $79(90.8 \%)$ & $309(18.40)$ & $64(79.0 \%)$ & $101(14.93)$ \\
\hline Chronic obstructive pulmonary disease & $43(49.4 \%)$ & $96(5.73)$ & $38(46.9 \%)$ & $84(4.45)$ \\
\hline Dyspnoea & $40(46.0 \%)$ & $77(4.60)$ & $39(48.1 \%)$ & $74(3.91)$ \\
\hline Cough & $17(19.5 \%)$ & $24(1.42)$ & $16(19.8 \%)$ & $20(1.06)$ \\
\hline Productive cough & $14(16.1 \%)$ & $21(1.24)$ & $8(9.9 \%)$ & $13(0.69)$ \\
\hline Nasopharyngitis & $12(13.8 \%)$ & $17(1.02)$ & $12(14.8 \%)$ & $14(0.73)$ \\
\hline Oropharyngeal pain & $10(11.5 \%)$ & $13(0.77)$ & $5(6.2 \%)$ & $7(0.37)$ \\
\hline Sputum discoloured & $9(10.3 \%)$ & $11(0.66)$ & $2(2.5 \%)$ & $2(0.11)$ \\
\hline Infections and infestations & $65(74.7 \%)$ & $180(10.73)$ & $54(66.7 \%)$ & $154(8.14)$ \\
\hline Lower respiratory tract infection & $18(20.7 \%)$ & $48(2.85)$ & $14(17.3 \%)$ & $33(1.75)$ \\
\hline Upper respiratory tract infection & $8(9.2 \%)$ & $12(0.73)$ & $9(11.1 \%)$ & $13(0.69)$ \\
\hline Gastrointestinal disorders & $32(36.8 \%)$ & $53(3.14)$ & $26(32.1 \%)$ & $47(2.48)$ \\
\hline Nausea & $10(11.5 \%)$ & $14(0.84)$ & $5(6.2 \%)$ & $6(0.33)$ \\
\hline Diarrhoea & $8(9.2 \%)$ & $10(0.58)$ & $10(12.3 \%)$ & $12(0.62)$ \\
\hline General disorders and administration site conditions & $23(26.4 \%)$ & $53(3.14)$ & $26(32.1 \%)$ & $47(2.48)$ \\
\hline Fatigue & $9(10.3 \%)$ & $16(0.95)$ & $9(11.1 \%)$ & $10(0.51)$ \\
\hline Musculoskeletal and connective tissue disorders & $21(24.1 \%)$ & $29(1.72)$ & $23(28.4 \%)$ & $33(1.75)$ \\
\hline Skin and subcutaneous tissue disorders & $17(19.5 \%)$ & $22(1.31)$ & $11(13.6 \%)$ & $11(0.58)$ \\
\hline Nervous system disorders & $15(17.2 \%)$ & $22(1.31)$ & $12(14.8 \%)$ & $17(0.91)$ \\
\hline Headache & $9(10.3 \%)$ & $12(0.73)$ & $10(12.3 \%)$ & $13(0.69)$ \\
\hline
\end{tabular}

Only events or system organ classifications with $\geqslant 10 \%$ incidence in at least one of the treatment groups are shown. A patient with more than one specific TEAE was counted once for that class. Data are presented as $\mathrm{n}(\%)$ or $\mathrm{n}$ (incidence rate per 100 days), with the incidence rate based on exposures of 61.32 AAT patient-years and 69.10 placebo patient-years. TEAE: treatment-emergent adverse events; AAT: $\alpha 1$-antitrypsin. ${ }^{\#}: \mathrm{n}=87 ;{ }^{\text {ๆ: }} \mathrm{n}=81$.

One 71-year-old patient in the AAT-treated group died during the treatment period, although this was not considered to be related to the study drug (arterial haemorrhage after 190 days of study). No deaths during the treatment period were reported in the placebo group.

Lung function was initially captured in the clinical protocol under the safety evaluation, because it was not thought to be sufficiently powered to detect an efficacy signal, owing to the small sample size and the relatively short duration of the study. At week 50 of the study, 53 AAT-treated patients had a mean FEV1 of $1.37 \mathrm{~L}$ (95\% CI 1.47-1.29 L), while 68 placebo patients had a mean FEV1 of $1.31 \mathrm{~L}$ (95\% CI 1.37-1.28 L; $\mathrm{p}=0.08$ ). Additional post hoc analyses of lung function results are presented in the supplementary material, supplementary table S4 and supplementary figures S1-S3.

\section{Immunogenicity}

In the AAT-treated group, $63 \%$ of patients developed anti-drug antibodies (ADAs) with no observed impact on safety or the plasma concentration of AAT (data not shown). The majority of the patients (90\%) had a titre below 1:32, and in most cases titres declined over time ( 1 year of treatment). No classical anaphylactic or hypersensitivity events were recorded. Within the AAT-treated group, if anything, the $\mathrm{ADA}+$ patients had lower event rates of withdrawals, SAEs and suspected hypersensitivity than the ADApatients (table 4).

\section{Discussion}

This is the first randomised placebo-controlled clinical trial to study the effects of inhaled AAT in ZZ-AATD COPD patients with a high risk for exacerbations. In this study population, AAT inhalation did not prolong the time to the first exacerbation after randomisation, nor to the second or third.

Inhalation of AAT represents an alternative to the i.v. route, offering easier and more efficient delivery of AAT directly to the lungs, enriching the lung epithelial lining fluid (ELF) with AAT levels at least three times higher than those achieved by i.v. administration $[14,15]$. This enhances the local antiprotease action of AAT, as well as potentially having a direct effect upon inflammation through inhibition of proteinase-driven events $[16,17]$ and binding to proinflammatory cytokines [18]. The rationale for the 
TABLE 4 Rates of adverse events with relation to antibodies to ADA

\begin{tabular}{|c|c|c|}
\hline \multirow[b]{2}{*}{$\mathrm{AE}$} & \multicolumn{2}{|c|}{ Rate per 100 days } \\
\hline & $A D A+{ }^{\#}$ & ADA- 7 \\
\hline All adverse events & 3.26 & 6.40 \\
\hline Leading to discontinuation & 0.12 & 0.90 \\
\hline Suspected hypersensitivity & 0.11 & 0.28 \\
\hline Serious adverse events & 0.11 & 0.36 \\
\hline Related & 0.65 & 2.44 \\
\hline All respiratory TEAEs & 1.56 & 2.71 \\
\hline All exacerbations & 0.78 & 1.11 \\
\hline
\end{tabular}

dose of AAT in the present study is based on studies showing that inhalation of twice-daily doses of $100 \mathrm{mg}$ AAT can increase ELF AAT levels from $0.28 \mu \mathrm{M}$ to $5.86 \mu \mathrm{M}$ [15]. It is assumed that in line with these results, a daily inhaled dose of $160 \mathrm{mg}$ of AAT would increase the AAT in the ELF to $\sim 4.4 \mu \mathrm{M}$, which is above the threshold of $1.7 \mu \mathrm{M}$ estimated to be protective in non-AAT-deficient patients and similar to the values $(2-5 \mu \mathrm{M})$ seen in healthy volunteers [19].

Fewer symptoms were observed in the first exacerbation. The nature of the exacerbation changed with AAT treatment, which suggests that AAT might influence the incidence or severity of dyspnoea, which is the cardinal exacerbation symptom in this patient cohort [7]. Patients perceive dyspnoea as a significant factor in their condition as it worsens and, conversely, perceive a decrease of dyspnoea as beneficial [6]. Interpretation of the cause and the implications of this observed symptom change during exacerbations need to be confirmed by a more focused study.

The data in table 2 reveal that not all changes in symptoms (as part of the Anthonisen criteria and classified as symptom-based exacerbations) resulted in treatment with a course of antibiotics or oral steroids, which would turn the episode from a symptom-based to an event-based moderate exacerbation. In part, this may have been related to the protocol design, which instructed that treatment of a moderate/ severe exacerbation was predominantly left to local medical practitioners or to patients who possessed a rescue package prescribed by the local physicians (either general practitioners or consultant physicians in the local clinic) if the patient felt that treatment was needed.

It is possible that daily electronic prompting of patients to record changes in respiratory symptoms in the previous $24 \mathrm{~h}$ may have had an effect on the patterns seen in our study compared with other studies. However, the patients included in the trial had to have a history of frequent exacerbations as an entry criterion. A previous study [12] that used the same Bronkotest as used in our study also reported that patients with AATD-related COPD had many untreated exacerbations. This suggests that over-reporting is not an issue and that prompting patients to report their symptoms if they had not reported by 11:00 $\mathrm{h}$ more likely highlights the real pattern of change in individual symptoms. Indeed, table 2 shows that many of the first exacerbations, classified by Anthonisen criteria, were not treated by oral steroids or antibiotics.

The use of an eDiary has highlighted several features previously unrecorded. Real-time data collection provides an opportunity (outside the scope of this manuscript) to show that the course of respiratory symptoms in our study population changes in many ways. This has implications for patient management. First, if respiratory symptoms recorded as baseline during a run-in period of the study become persistently lower following a treated exacerbation while the patient is on study medication, does this imply that the baseline symptoms recorded at run-in have to be reset? Second, does an exacerbation of a symptom with a high score in the Bronkotest that does not return to its baseline score indicate an inadequate typed exacerbation or inadequate length of therapy or a progression of disease features? Both scenarios are rarely considered in the long-term management of AATD-related COPD.

Serum antibody development of ADAs occurred in 63\% of participants treated with inhaled AAT. The clinical relevance of these antibodies is, however, unclear. First, there was no association with reported adverse events or withdrawal rate. Second, it has been reported that very sensitive ADA assays, such as those used in the present study, may contribute to the high ADA incidence detected [20]. Furthermore, the titres fell with time, indicating that the seropositivity was not a cumulative phenomenon.

The safety profile was also influenced by a slight imbalance between treatment groups in terms of higher baseline age and disease severity in the AAT-treated group (table 1). Although a placebo was necessary for 
the study, it remains possible that the nebulised placebo (saline+phosphate) could have had an impact on symptoms. It should be noted that the safety profile of the two groups became similar after the first year of the study, once improvements in device handling had been made to the nebulisation process, which resolved patient complaints about long nebulisation time.

The RAPID study also reported a higher annual rate of SAEs and exacerbations in the AAT-treated than in the placebo group (57 versus 45 and 1.7 versus 1.4, respectively) [10]. In respiratory disease, one of the most common negative reactions to inhaled drugs is a reduction in lung function. This reduction is often related to potential immunogenicity reactions and hyperreactivity reactions and/or bronchospasm. In our study, however, the AAT-treated group did not experience a reduction in lung function at the end of the study, which decreases the possibility that they may have experienced any hyperreactivity reaction. Unexpectedly, the rapid increase in FEV1 after 4 weeks of inhaled AAT and the decline of FEV1 4 weeks after stopping AAT inhalation (supplementary figures S1 and S3) suggest that AAT inhalation exerts an early anti-inflammatory effect on the airways. The reason for divergence of change in FEV1 and the level of airflow obstruction between treatment arms is currently unknown and warrants a new study with decline in FEV1 as the primary outcome parameter.

There are several potential limitations to the study. At the time the study was designed and the primary endpoint of time to first moderate or severe exacerbation was chosen, the phenotype of frequent exacerbators was not yet defined. It might be that in this patient population, a 1-year study was too short to observe a change in the rate of exacerbation. As published subsequently by HuRsT et al. [21], two-thirds of COPD patients without AATD who have this phenotype continue to experience frequent exacerbations for 3 years, suggesting that a 2-3 year study may be more appropriate in AATD.

We conclude that in AATD patients with severe COPD and frequent exacerbations, AAT inhalation had no effect on the time to first exacerbation during treatment for 50 weeks. After improvements were made to the handling of the AAT and nebuliser, the safety profile was similar between groups and resembled the rates previously reported in i.v. AAT studies.

Author contributions: J. Stolk was involved in the design of the study, was principal investigator for Leiden University Medical Centre and collected data from study patients. He was involved in data analysis and interpretation and wrote the article in collaboration with N. Tov, P. Fernandez and R.A. Stockley. N. Tov was involved in the design of the study and oversight of study conduct. He was involved in data analysis and interpretation, and wrote the article in collaboration with other co-authors. K.R. Chapman was principal investigator for his site and collected data from study patients. He was involved in data analysis and contributed to the article. P. Fernandez was involved in the design of the study. He analysed the diary cards and was involved in data analysis and interpretation and contributed to the article. W. MacNee was principal investigator for his site and collected data from study patients. He was involved in data analysis and contributed to the article. N.S. Hopkinson was principal investigator for his site and collected data from study patients. He was involved in data analysis and contributed to the article. E. Piitulainen was principal investigator for her site and collected data from study patients. She was involved in data analysis and contributed to the article. N. Seersholm was principal investigator for his site and collected data from study patients. He was involved in data analysis and contributed to the article. C.F. Vogelmeier was principal investigator for his site and collected data from study patients. He was involved in data analysis and contributed to the article. R. Bals was principal investigator for his site and collected data from study patients. He was involved in data analysis and contributed to the article. G. McElvaney was principal investigator for his site and collected data from study patients. He was involved in data analysis and contributed to the article. R.A. Stockley was involved in the design of the study, was principal investigator for his site, and collected data from study patients. He analysed the diary cards and was involved in data analysis and interpretation and wrote the article in collaboration with N. Tov, P. Fernandez, and J. Stolk.

Conflict of interest: J. Stolk reports personal fees for consultancy from Kamada Ltd, during the conduct of the study, and grants from CSL Behring, outside the submitted work. N. Tov reports personal fees for consultancy from Kamada Ltd, during the conduct of the study, and is an employee of Kamada Ltd, outside the submitted work. K.R. Chapman reports personal fees for consultancy from Kamada Ltd, during the conduct of the study, and grants from CSL Behring and Grifols, outside the submitted work. P. Fernandez reports consultancy fees from Kamada Ltd, during the planning, design, conduct and reporting of the study. W. MacNee reports patient recruitment fees from Kamada Ltd, during the conduct of the study; and grants and personal fees from Pfizer and GlaxoSmithKline, and personal fees from Boehringer Ingelheim, AstraZeneca, Novartis, Zambon and Chiesi, outside the submitted work. N.S. Hopkinson has nothing to disclose. E. Piitulainen has nothing to disclose. N. Seersholm has nothing to disclose. C.F. Vogelmeier reports grants and personal fees from AstraZeneca, Boehringer Ingelheim, GlaxoSmithKline, Grifols and Novartis, and personal fees from CSL Behring, Chiesi, Menarini, Mundipharma, Teva and Cipla, outside the submitted work. R. Bals reports grants from Kamada Ltd, during the conduct of the study; and grants from BMBF, DFG, Schwiete Stiftung, Sander-Stiftung and Boehringer Ingelheim, and personal fees for advisory board work and travel to meetings from GlaxoSmithKline, CSL Behring, Boehringer Ingelheim, Grifols, AstraZeneca and Novartis, outside the submitted work. G. McElvaney reports personal fees for advisory board work from CSL Behring, grants and personal fees for advisory board work from Grifols, and grants from Chiesi, outside the submitted work. R.A. Stockley reports personal fees for advisory board membership from Kamada Ltd, during the conduct of the study; personal fees for advisory board membership and lectures from AstraZeneca, personal fees for advisory board membership from Medimmune, Almirall, Baxter, Chiesi and Polyphor, personal fees for lectures from Nycomed and Takeda, and personal fees for advisory board membership, lectures and travel to meetings from Boehringer Ingelheim and CSL Behring, outside the submitted work. 
Support statement: Kamada Ltd was the sponsor of the study and delivered all data obtained during the clinical trial and all statistical analyses to all authors. Funding information for this article has been deposited with the Crossref Funder Registry.

\section{References}

1 Eriksson S. Pulmonary emphysema and alpha1-antitrypsin deficiency. J Intern Med 1964; 175: 197-205.

2 Alpha-1-Antitrypsin Deficiency Registry Study Group. Survival and FEV1 decline in individuals with severe deficiency of alpha1-antitrypsin. Am J Respir Crit Care Med 1998; 158: 49-59.

3 Needham M, Stockley R. Exacerbations in $\alpha 1$-antitrypsin deficiency. Eur Respir J 2005; 25: 992-1000.

4 Dowon LJ, Guest PJ, Stockley RA. Longitudinal changes in physiological, radiological, and health status measurements in $\alpha 1$-antitrypsin deficiency and factors associated with decline. Am J Respir Crit Care Med 2001; 164: 1805-1809.

5 Ejiofor SI, Stolk J, Fernandez P, et al. Patterns and characterization of COPD exacerbations using real-time data collection. Int J Chron Obstruct Pulmon Dis 2017; 12: 427-434.

6 Anzueto A, Miravitlles M. Pathophysiology of dyspnea in COPD. Postgrad Med 2017; 129: 366-374.

7 Anthonisen NR, Manfreda J, Warren CP, et al. Antibiotic therapy in exacerbations of chronic obstructive pulmonary disease. Ann Intern Med 1987; 106: 196-204.

8 Wewers MD, Casolaro MA, Sellers SE, et al. Replacement therapy for alpha 1-antitrypsin deficiency associated with emphysema. N Engl J Med 1987; 316: 1055-1062.

9 Parr DG, Dirksen A, Piitulainen E, et al. Exploring the optimum approach to the use of CT densitometry in a randomised placebo-controlled study of augmentation therapy in alpha 1-antitrypsin deficiency. Respir Res 2009; 10: 75 .

10 Chapman KR, Burdon JG, Piitulainen E, et al. Intravenous augmentation treatment and lung density in severe alphal antitrypsin deficiency (RAPID): a randomised, double-blind, placebo-controlled trial. Lancet 2015; 386: 360-368.

11 Quanjer PH, Cooper B, Ruppel GL, et al. Defining airflow obstruction. Eur Respir J 2015; 45: 561-562.

12 Vijayasaratha K, Stockley RA. Reported and unreported exacerbations of COPD: analysis by diary cards. Chest J 2008; 133: 34-41.

13 Thompson BR, Johns DP, Bailey M, et al. Prediction equations for single breath diffusing capacity DLCO (TLCO) in a middle aged Caucasian population. Thorax 2008; 63: 889-893.

14 Hubbard RC, McElvaney NG, Sellers SE, et al. Recombinant DNA-produced alpha 1-antitrypsin administered by aerosol augments lower respiratory tract antineutrophil elastase defenses in individuals with alpha 1-antitrypsin deficiency. J Clin Invest 1989; 84: 1349-1354.

15 Hubbard RC, Brantly ML, Sellers SE, et al. Anti-neutrophil-elastase defenses of the lower respiratory tract in $\alpha 1$-antitrypsin deficiency directly augmented with an aerosol of $\alpha 1$-antitrypsin. Ann Intern Med 1989; 111: 206-212.

16 Petrache I, Fijalkowska I, Zhen L, et al. A novel antiapoptotic role for alpha1-antitrypsin in the prevention of pulmonary emphysema. Am J Respir Crit Care Med 2006; 173: 1222-1228.

17 Petrache I, Fijalkowska I, Medler TR, et al. $\alpha-1$ antitrypsin inhibits caspase-3 activity, preventing lung endothelial cell apoptosis. Am J Pathol 2006; 169: 1155-1166.

18 Bergin DA, Reeves EP, Meleady P, et al. Alpha-1 antitrypsin regulates human neutrophil chemotaxis induced by soluble immune complexes and IL-8. J Clin Invest 2010; 120: 4236-4250.

19 Franciosi AN, McCarthy C, McElvaney NG. The efficacy and safety of inhaled human alpha-1 antitrypsin in people with alpha-1 antitrypsin deficiency-related emphysema. Expert Rev Respir Med 2015; 9: 143-151.

20 Song S, Yang L, Trepicchio WL, et al. Understanding the supersensitive anti-drug antibody assay: unexpected high anti-drug antibody incidence and its clinical relevance. J Immunol Res 2016; 2016: 3072586.

21 Hurst JR, Vestbo J, Anzueto A, et al. Susceptibility to exacerbation in chronic obstructive pulmonary disease. $N$ Engl J Med 2010; 363: 1128-1138. 